PostGRAD. MED. J. (1966), 42, 207

Current Surveys

\title{
THE DIAGNOSIS AND SURGICAL ASSESSMENT OF CARCINOMA
}

\section{OF THE BRONCHUS}

\author{
H. C. NoHL-Oser, M.A., D.M., F.R.C.S. \\ Consultant Thoracic Surgeon to Harefield, Hillingdon and West Middlesex Hospitals, Middx.
}

AT PRESENT surgical treatment of lung cancer offers the best prospect of cure. The earlier the resection the greater the chances of cure. Delay in the diagnosis occurs because many of these tumours are asymptomatic for a long time or simulate other, commoner conditions. This paper emphasises the diagnostic methods available and the circumstances in which their application is most likely to be fruitful.

\section{Presentation}

Much of the difficulty in the early diagnosis of cancer of the lung lies in the fact that the clinical picture is so variable.

Carcinoma of the lung may present itself in one of the following ways:

1. Symptomatically by the well-known symptoms of cough, bloodstained sputum, shortness of breath, loss of weight, pain in the chest.

2. By a febrile chest illness: a recent history of "simple" pneumonia which does not resolve or recurs after initial response to antibiotic treatment. In this category the so-called "virus pneumonia" must be included.

3. By unusual manifestations.

4. As an asymptomatic lesion discovered on routine chest radiography.

1. Symptoms: Lung cancer occurs at any age but is more common after the age of 40 . It is no longer a rare diselase in women. The association between smoking and bronchial oarcinoma should be remembered. Because many of the patients are smokers, the history of cough, the most common presenting symptom, must be enquired into with detail. Haemoptysis, although an alarming symptom, occurred as the first symptom in only $14 \%$ of 1,200 cases (Salzer, Wenzl, Jenny and Stangl, 1952). It should never be ignored and must be investigated by bronchoscopy in every patient over the age of 40 , even though the chest radiograph shows no abnormality. Chest pain is not a late manifestation, as is usually presumed but is often fleeting, frequently described as mere discomfort; it should never be attributed to "rheumatism" or "fibrositis". Pain in the chest warrants radiological examination. Old men are often dyspnoeic because of bronchitis and emphysema and dyspnoea is therefore all too often dismissed as a sign of bronchitis or just age. Shortness of breath was the first symptom in $21 \%$ of Salzer's cases and should therefore always be followed by radiological examination. Lassitude is another vague symptom which ranks high in the list of early symptoms of lung cancer, while loss of weight does not invariably mean inoperability.

2. Febrile Chest Illness: Sooner or later the growth leads to bronchial obstruction which causes atelectasis of the involved segment or lobe. The symptoms and signs then may simulate pneumonia, pleurisy, lung abscess, bronchiectasis or empyema. The history of "flu" or a "touch of pneumonia," frequently treated with antibiotics with initial response, is a common story. Every patient recovering from a febrile chest illness should have a radiological examination and should not be considered as having recovered until the lung fields are absolutely clear. "Uno resolved pneumonia" is a dangerous diagnosis this should suggest the possibility of neoplasmo 3. Unusual Manifestations: These modes of presentation often simulate disease affecting other parts of the body. The commonest is hypertrophic pulmonary osteoarthropathy, causing pain and swelling in the joints and is therefore often diagnosed as rheumatism or arthritis. Clubbing of the fingers and toes can usually be detected, but may be absent. In long-standing cases radiography of the long bones, showing subperiosteal bone deposition, confirms this condition. Carcinomatous neuromyopathy manifests itself bilaterally and typically involves the proximal muscles of the limbs. These features distinguish it from brain or spinal metastases. Various types have been described by Brain and Henson (1958):

(a) Cortico-cerebellar degeneration.

(b) Mixed forms.

(c) Sensory neuropathy.

(d) Peripheral neuropathy.

(e) Neuromuscular disorders.

Myasthenic myopathy with poor response to neostigmine has also been reported (Rooke, Eaton, Lambert and Hodgson, 1960). The symptoms may develop concurrently with the tumour or may precede the clinical manifestation of the growth by long periods. It has even appeared after successful removal of the tumour.

Patients with an apical tumour complain of pain in the shoulder, often radiating down the arm. The history of prolonged physiotherapy with no relief of their symptoms is not un- 


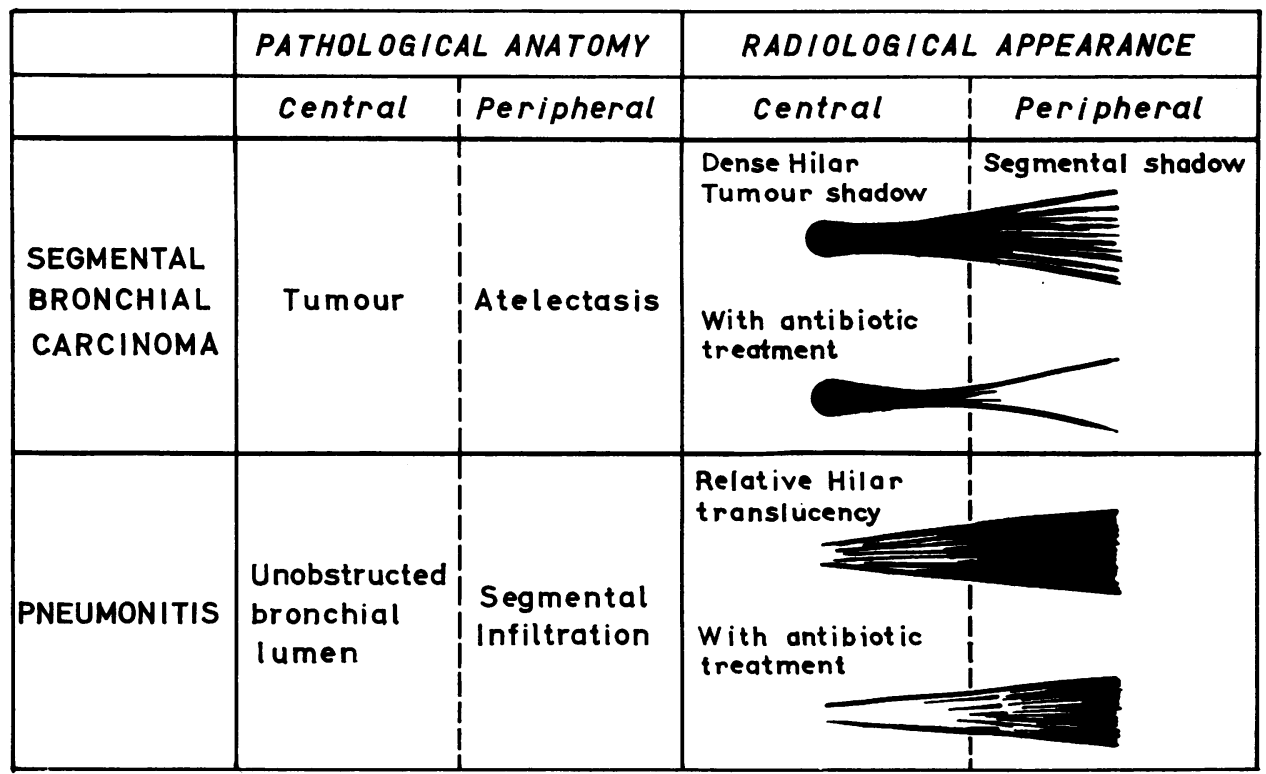

FIG. 1.-Diagrammatic representation of the main features which distinguish pneumonitis from bronchial carcinoma radiologically. (Modified from F. Olbert, Med. Klin., 57, 429, 1962).

common. These apical tumours are not easily seen on the usual P.A. (postero-anterior) chest films as they often involve only the pleura. An A.P. film of the apex or tomography may detect the lesion. Many of these tumours, contrary to the usual opinion, can be resected with relief of the severe pain (Dontas, 1957).

4. Asymptomatic lesions discovered on routine chest radiography. The majority of these lesions are solitary circumscribed pulmonary shadows, often described as "coin lesions". Over $50 \%$ of these solitary shadows are malignant (Good and Wilson, 1959). They pose a most difficult diagnostic problem. In some cases tomography may be of help. Malignancy can be suspected when the shadow shows lobulation or has an indefinite edge with radiating striae ("orab feet") extending into the surrounding lung parenchyma. Calcification in the lesion does not rule out malignancy (Davis, Peabody and Katz, 1956; O'Keefe, Good and McDonald, 1957). O'Keefe found calcification to be present in $13.9 \%$ of malignant lesions in a series of 207 solitary nodules removed by operation. Tomography may demonstrate a cavity with a thick irregular wall due to central necrosis of the tumour. The knowledge that a solitary round shadow has been present for a year or more without increase in size is no guarantee of its innocence. Davis and others (1956) record 25 such cases which had been under observation for 1-8 years. In my opinion it is indefensible to watch these lesions in patients over the age of 40 , nor is it wise to try the effect of antituberculosis chemotherapy as even tuberculomas will often not alter on prolonged treatment. As there is no way of distinguishing the benign from the malignant lesion it is far safer to advise excision in every case.

\section{The Diagnosis and Surgical Assessment}

Once the suspicion of lung cancer has been raised, a series of methodical investigations have to be carried out in a Thoracic Unit, until the diagnosis has been established or excluded.

Radiology. The majority of cases of bronchial carcinoma show radiological shadows, either the tumour mass itself or the changes resulting from bronchial obstruction are seen. Besides the usual P.A. film of the chest, a lateral radiograph is essential. Not only will the lateral film help in the localisation of the shadow, but lesions in the left lower lobe, hidden behind the heart shadow, will become obvious. The centrally situated bronchial carcinoma causes a simple atelectasis only when there is complete bronchial obstruction by the tumour. In the majority of cases, however, sputum retention beyond the tumour stenosis leads to segmental or lobar infective infiltration which may result in abscess formation. Segmental collapse causes a sharply defined homogeneous, wedge shaped shadow. Some of the distinguishing appearances between atelectasis caused by inflammatory conditions and those due to neoplasm, have recently been described by Olbert (1962) and are illustrated in diagrammatic form in Fig. 1. In cases where segmental or lobar collapse is caused by a carcinoma, the area near the hilum shows a dense shadow which corresponds to the tumour. The thin edge of the wedge is often round rather than pointed. In cases of pneumonitis, however, 
in which the bronchial lumen is relatively unobstructed, the atelectatic shadow is more dense at the periphery, while towards the hilum the wedge becomes translucent. These differences become even more marked when treatment with broad-spectrum antibiotics and physiotherapy is instituted. The atelectatic shadow due to carcinoma will shrink progressively and the periphery may become more translucent but the hilar density will remain and become more obvious. In the case of chronic pneumonia, however, there is no tendency to shrinkage of the atelectatic wedge, but a progressive translucency ocurs beginning at the hilum, while the periphery is the last to clear. These observations, following a trial of antibiotic treatment, have been of diagnostic value in those difficult cases where the differential diagnosis could not be established for certain by the other methods of investigations about to be described. Prolonged periodic review, however, is dangerous.

Radiology has its limitations: a carcinoma situated in a main bronchus will not show radiological changes until total obstruction occurs. In such cases auscultation of the chest will often reveal a persistent, localised, expiratory rhonchus, frequently associated with diminished or absent breath sounds. It is in these patients that obstructive emphysema is likely to be demonstrated on radiographs taken on expiration rather than during the routine inspiratory phase.

Tomography. Tomography either by P.A. or lateral views is frequently used for further definition of the tumour shadow. It will demonstrate especially well the tumour edge and in cases of abscess formation will show the thick irregular wall due to tumour necrosis. Tomography confined to these two planes is not reliable in showing bronchial stenosis, as the bronchi are not quite parallel with the plane of the tomograph. A patent bronchus might appear to be narrowed where it transects the level at which the film is focussed.

Bronchography is rarely indicated.

Sputum Cytology. This examination requires considerable skill and can be time-consuming, especially if stains involving fixation are used (Papanicolaou, 1942, 1949). Hinson and Kuper (1963), using the methylene blue preparation of unfixed material (Schuster, 1955) have shown that very satisfactory results can be obtained by searching 2 slides for 5 minutes each. Positive results were obtained in $60 \%$ of cases in 229 patients. Furthermore, the necessary proficiency can be acquired within approximately three months. This method of investigation is especially of value in peripheral tumours beyond bronchoscopic vision; they found positive results in over $60 \%$ of these cases. These results confirm the findings of Jennings and Shaw (1953). The incidence of false positive results is under $2 \%$ and usually occurs when there is squamous metaplasia due to long standing bronchiectasis or tuberculosis cavities. Sputum cytology should be employed before bronchoscopy, as the instrumentation may cause shedding of tiny fragments of mucosa which may give false positive results.

Bronchoscopy. This is the next step in the investigation. This procedure is much better carried out under general anaesthesia, because not only is it less unpleasant to the patient but the complete paralysis of the patient also allows better visualization, especialy of the segmental divisions of the left upper lobe. After exact radiological localization it is often possible to obtain a positive result by a blind biopsy with the angled biopsy forceps from the appropriate segmental bronchus, even when no actual growth is seen. This blind procedure is safe provided that the biopsy forceps can be withdrawn without much traction after closure of the jaws.

Bronchoscopy will also help in the assessment of technical operability; the following findings indicate that resection is impossible: paralysis of the vocal cords, involvement of the lower end of the trachea or the origin of a main bronchus, a rigid and widened carina.

Bronchoscopy, however, has its limitations. The field of possible vision is indicated in Fig. 2. In 211 patients in whom the growth was resected, a positive bronchial biopsy was obtained in 107 $(50 \%)$. If patients with more advanced growth@ are included, positive biopsies can be expecte in $70 \%$ of cases.

It has been said with some justification, that a negative bronchoscopy is even more dangerous than no bronchoscopy at all, as it lulls the physician into false sense of security.

Pleural Biopsy. The recognition of malignant cells in pleural fluid is difficult, as pleural mesothelial cells are easily mistaken for neoplastic ones. A pleural biopsy using the Abram's needle (Abrams, 1958) gives more reliable results. A positive biopsy is likely to be obtained in $60-70 \%$ of cases with malignant effusions (Mestiz, Purves and Pollard, 1958; Hanson and Phillips, 1962). A positive biopsy means inoperability in my view, although some authors have advised pleuropneumonectomy.

Mediastinoscopy. This procedure, which was first practised by Carlens (1959), is useful in establishing the diagnosis in any form of hilar glandular enlargement and in assessing operability in otherwise seemingly operable cases of carcinoma of the bronchus (Nohl-Oser, 1965). This investigation has really superseded scalene node biopsy (Daniels, 1949), as it allows palpation of the mediastinum and enables one to obtain a biopsy very much nearer to the lung root. In 36 of 76 patients with lung cancer a positive mediastinal biopsy was obtained, in 15 of which the bronchoscopy was negative. Mediastinoscopy will save many patients from unnecessary thoracotomies. In the experience of most authors (Reynders, 1963; van der Schaar and van Zanten, 

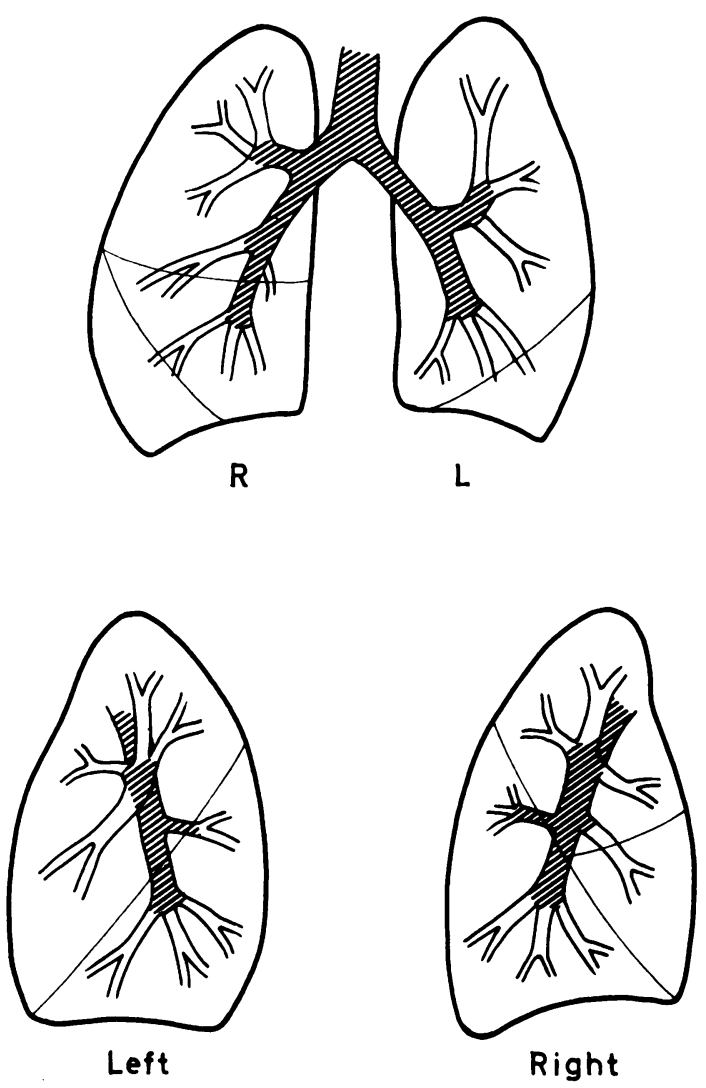

FIG. 2.-Diagrammatic drawing of the right and left bronchial trees with the bronchoscopic visual field shaded.

$1965)$ it is most unlikely that the growth is resectable if the mediastinal biopsy is positive. Oat celled carcinoma is known to spread rapidly to the mediastinal lymph nodes (Nohl, 1962) and for this reason mediastinoscopy is especially recommended in all patients in whom the bronchial biopsy reveals this type of growth.

Exploratory Thoracotomy. There remain the few cases where every investigation has failed to confirm the suspicion of malignancy. It is in these cases where it is ultimately necessary to proceed to thoracotomy, provided, of course, that the general condition and the respiratory reserve of the patient are satisfactory. It should however be pointed out that it is not always easy to be certain of the pathology even when the chest is open. In cases of doubt one is then compelled to proceed with resection and await the final pathological report.

Summary

The variable clinical picture of lung cancer often makes the diagnosis difficult. First of all, it is important to suspect the presence of malignancy of the lung. This suspicion must then be pursued to a prompt conclusion by a series of systematic investigations, which cannot be made at the bedside or in the general practitioner's surgery. Radiological appearances which are of help in the differential diagnosis and the limitation of radiology are discussed. Periodic radiological review of a possibly malignant lesion is fatal. Bronchoscopy must never be omitted, but negative findings should not give the physician a false sense of security. Examination of the sputum for malignant cells by a trained cytologist is valuable in those cases where the growth lies beyond bronchoscopic vision. Mediastinoscopy has been of value in the diagnosis of hilar lymphadenopathy and in the assessment of operability. Finally, one should not hesitate to advise an exploratory thoracotomy in the few cases which remain unconfirmed.

I wish to thank Dr. A. H. James for helpful criticism and Miss K. J. Graham, Medical Artist, Hillingdon Hospital, for the diagrams.

\section{REFERENCES}

Abrams, L. D. (1958): A Pleural Biopsy Punch, Lancet, i, 30.

Brain, R. W., and Henson, R. A. (1958): Neurological Syndromes Associated with Carcinoma, the Carcinomatous Neuropathies, Lancet, ii, 971.

Carlens, E. (1959): Medliastinoscopy, a Method for Inspection and Tissue Biopsy in the Superior Mediastinum, Dis. Chest, 36, 343.

Daniels, A. C. (1949): A Method of Biopsy Useful in Diagnosing Certain Intrathoracic Diseases, Dis. Chest, 16, 360.

Davis, E. W., Peabody, J. W., and Katz, S. (1956): The Solitary Pulmonary Nodule, J. thorac. Surg., 32, 728 .

DontaS, N. S. (1957): The Pancoast Syndrome, Brit. J. Tuberc., 51, 246.

Good, C. A., and WiLson, T. W. (1959): The Solitary Circumscribed Pulmonary Nodule, J. Amer. med. Ass., 166, 210.

Hanson, G., and Phillips, T. (1962): Pleural Biopsy in Diagnosis of Thoracic Disease, Brit. med. J., ii, 300.

HiNSON, K. F. W., and KUPER, S. W. A. (1963): The Diagnosis of Lung Cancer by Examination of Sputum, Thorax, 18, 350.

Jennings, R. C., and Shaw, K. M. (1953): The Value of Cytological Examination of the Sputum in the Diagnosis of Carcinoma of Bronchus, Thorax, 8, 288.

Mestiz, P., Purves, M. J., and Pollard, A. C. (1958): Pleural Biopsy in the Diagnosis of Pleural Effusion, a Report of 200 Cases, Lancet, ii, 1349.

NoHL, H. C. (1962): The Spread of Carcinoma of the Bronchus, London: Lloyd Luke.

NOHL-OSER, H. C. (1965): Mediastinoscopy, Brit. med.J., i, 1167.

O'KeEFE, M. E., Good, C. A., and McDonald, J. K. (1957): Calcification in Solitary Nodules of the Lung, Amer. J. Roentgenol., 77, 1023. 
OlBERT, F. (1962): Wandlungen der Klinischen Problematik des Bronchus Karzinoms im letzten Jahrzehnt, II Die röntgenologische Erfassung von Verdachtsfällen, Med. Klin., 57, 428.

Papanicolaou, G. N. (1942): New Procedure for Staining Vaginal Smears, Science, 95, 438.

Papanicolaou, G. N. (1949): A Survey of the Actualities and Potentialities of Exfolliative Cytology in Cancer Diagnosis, Ann. intern. Med., 31, 661.

REYNDERS, H. (1963): Mediastinoscopie, Thesis, Amsterdam.
ROOKe, E. D., EATON, L. M., LAMBert, E. H., and HoDGson, C. H. (1960): Myasthenia and Malignant Intrathoracic Tumour, Med. Clin. N. Amer., 44, 977.

Salzer, G., Wenzl, M., Jenny, R. H., and Stangl, A. (1952): Das Bronchus-carcinom, Wien: Springer Verlag.

SCHAAR, P. J.v.d., and ZANTEN, M. E.v. (1965): Experience with Mediastinoscopy, Thorax, 20, 211.

SCHUSTER, N. (1955): Cancer Cells in the Sputum, Tubercle., 36, 350.

\title{
THE TREATMENT OF CARCINOMA OF THE BRONCHUS
}

\author{
JoHN W. JACKSON, M.Ch., F.R.C.S. \\ Harefield Hospital, Middlesex
}

In 1933, the first successful pneumonectomy for carcinoma of the lung was carried out. This patient-a doctor-lived for almost thirty years without recurrence, and died in 1963 at the age of seventy-nine. Not all the early cases of this type of surgery had such a successful course, and it was not until endotracheal anaesthesia, antibiotics and blood became readily available that lobectomy and pneumoneotomy became routine procedures in the treatment of lung cancer. In the immediate post-war period, the risks of operation were acceptable, but the long-term results were still disappointing, and British surgeons, who have always taken an aggressive attitude towards this disease, had a chance to take a second look at their results so as to discover the reasons for their apparent failure and develop new techniques which could extend the scope of the operation and at the same time bring about improvements in the long-term survival rate.

An important step forward was made when Allison showed that it was possible to take the pulmonary vessels inside the pericardium. Up to this time, it had been usual to tie the pulmonary artery first, as it was felt that this might reduce the amount of blood lost by pooling in the lung. Aylwin (1951) working on the same unit, emphasised the importance of early, or primary, vein ligation as a means of reducing the risks of blood stream dissemination of tumour due to manipulation of the lesion at the time of operation, and showed how this procedure was associated with a significant reduction in the number of early post-operative recurrences. With the same object in mind, Brock and Whytehead (1955) applying the principles of cancer surgery as carried out elsewhere in the body, described what they termed "radical resection", in which the affected lung, with its fascial connections and lymphatic field is removed in one block. This work was largely based on earlier anatomical studies of the lymphatics of the lungs and mediastinum, which did not necessarily always take account of the direction of spread of the tumour in the lymphatic system. Nohl (1956), in a study of clinical and operative material, made careful note of the gland involvement from tumours in different situations, and showed that in many cases, particularly where the disease is in an upper lobe, lobectomy provides as radical a cancer removal as pneumonectomy; thus confirming the experience of Belcher (1956) who has been a firm exponent of lobectomy as a means of removing cancer because it causes less respiratory disability and allows a higher proportion of patients to continue in their former employment. Price Thomas (1959) and Jones (1959) extended the advantages of lobectomy by carrying out sleeve resections of the main bronchi with re-anastomosis of the lower lobes to the trachea, conserving functioning lung that would otherwise be unnecessarily removed by pneumonectomy. This is particularly important in the older age groups and those with poor respiratory reserve, for whom pneumonectomy carries a greatly increased mortality. Oocasionally it has been possible to extend the principles of sleeve resection to the pulmonary artery.

There can be no doubt that the more radical procedures give the best chance of cancer cure, yet nobody at the time of operation can judge which patient will or will not have a long-term survival apparently free from disease. There are strong reasons for treating the individual patient rather than the disease. Analysis of the results of operation produce two apparently paradoxical findings. Firstly, with earlier diagnosis and earlier surgery, there has not been an increase in the percentage of long-term survivors. This 